\title{
The Application of Wearable Technologies to Improve Healthcare in the World's Poorest People
}

\author{
James A. Levine \\ Obesity Solutions, Mayo Clinic, 13400 E Shea Blvd, Scottsdale, AZ 85259 and Arizona State University, Fulton School of Engi- \\ neering, Tempe, Arizona, USA \\ Email: Levine.james@mayo.edu
}

How to cite this paper: Levine, J.A. (2017) The Application of Wearable Technologies to Improve Healthcare in the World's Poorest People. Technology and Investment, 8, 83-95. https://doi.org/10.4236/ti.2017.82007

Received: February 23, 2017

Accepted: May 23, 2017

Published: May 26, 2017

Copyright $\odot 2017$ by author and Scientific Research Publishing Inc. This work is licensed under the Creative Commons Attribution International License (CC BY 4.0).

http://creativecommons.org/licenses/by/4.0/

\begin{abstract}
Wearable technologies could be influential for improving healthcare in underserved areas. Wearable sensors are straightforward to develop, have low production costs and the methods for processing high volumes of data are advanced. Here we examine the application of wearable technology for improving health in three poverty-related scenarios. The first is for diabetes prevention in Mexico City. The second is for medical care in the Kibera slum in Nairobi and the third is for the delivery of "health kits" to refugees. The analysis affirms that investment is worthwhile in mass scalable wearable technologies directed at the half of the world's population who live in poverty.
\end{abstract}

\section{Keywords}

Wearable Technology, Consumer Behavior, Poverty, Entrepreneurship

\section{Introduction}

Overall, the wearable market is expected to increase substantially. Wearable technologies are technology devices that are worn by a person and frequently include tracking information that is relevant to health and fitness. Wearable technologies are currently a $\$ 1$ billion business and estimated to grow more than five-fold in the next few years. A Transparency Market Research report estimated that the world wearable market was $\$ 750$ million in 2012 and expected it to increase to $\$ 5.8$ billion by 2018 . Some consider this to be an under-estimate; Juniper Research predicted that worldwide wearable technology market would grow from $\$ 1.4$ billion in 2013 to $\$ 19$ billion by 2018 . The market is primed for expansion. Advantages of wearable technologies include that they are mass scalable, possesses many functions, deliver high volumes of potentially high quality 
data and can be disseminated wherever there are people. For these reasons, one large potential opportunity is for wearable sensing systems to improve the lives of the world's poor.

Half of the world's population, about 3 billion people, lives in poverty on less than $\$ 2.50$ /day; a third are children. $11 / 2$ billion people live in extreme poverty, on less than $\$ 1.25 /$ day. UNICEF asserts that 22,000 children die each year from poverty [1] [2] [3] [4].

In this paper, the application of wearable technologies for improving the health of the world's poor is examined.

\section{Wearable Technologies Have Come of Age; from Health Sensing through Drug Delivery}

The wearable technology market has matured to become applicable to poverty. There are several reasons.

\subsection{Wearable Device Design and Manufacture}

Prefabrication and fabrication of wearable devices is relatively straightforward and inexpensive. Software programs enable prefabrication of electronic circuits and physical casings to be designed and tested rapidly and inexpensive. The advancement in printable circuitry and printable three-dimensional structures enables prototypes to be produced quickly and at low cost. Once prototypes have been tested, the ability to convert early prototypes into advanced prototypes is also simple and also cost effective. Once the technology is fully tested and validated, mass production is simple and fast too. Thus the chain-of-production from concept to production has been greatly shortened with respect to wearable sensors.

\subsection{The Capability of Wearables Has Expanded}

The array of sensing and intervention modalities that can be integrated into a wearable technology has expanded enormously. A few years ago the technological capabilities of wearable sensors were limited; for example, resin-embedded uniaxial accelerometers were used to measure movement or glass coated thermistor couples were used to measure a person's surface skin temperature. Currently, smart triaxial accelerometers with self-regulating gravitational unit settings are available in inexpensive silicon chip integrated circuits. Gyroscopes are similarly embedded into chips and temperature and barometric pressure are readily measured using integrated circuit technology, too. EKG signals can be captured, as can respiratory patterns and even gaseous concentrations (which are potentially important for measuring inspiratory air quality). Intradermal and transdermal skin patches enable blood values to be continuously monitored such as glucose and electrolytes, which are important in diabetes and hydration. Skin patches can also sense the UV exposure of an individual to help prevent skin malignancy. Also enzyme-linked immunosorbent assays (ELISA's) can be integrated into wearables to measure infection exposure and lastly, transdermal 
patches can be used to deliver medications and vaccinations. These are only a few examples of different modalities that can be achieved using wearable sensors. Multiple sensors, medication patches and access to cloud based electronic medical records can be combined to form Integrated Wearable Health Kits that assess a person's medical needs and institute treatments. A simple example of an Integrated Wearable Health Kit would be where intradermal glucose is measured using a skin patch at the same time as body movement is measured using a wearable integrated circuit in a patient with Type I Diabetes. Through these multiplexed measures the algorithm signals the wearable insulin pump so that insulin doses can be adjusted to avoid episodes of hypoglycemia (low blood sugar). Thus, the array of wearable technologies has advanced so that multiple elements of human health can be improved upon.

\subsection{Well-Trained Workforce}

More students are being trained through college technology programs to exploit these types of technologies. Most major universities have centers of innovation and entrepreneurship. These centers encourage trainees of different levels to engage in technology development and entrepreneurship. There is a pipeline of developers emerging into the wearable market so that as the market expands rapidly as it is predicted to do in the next few years, there will be a well-trained workforce to take advantage of the greater need and opportunity.

\subsection{Large Volumes of Data Can Be Analyzed}

The knowhow exists to store and analyze large quantities of data. The methodologies have greatly advanced with respect to data storage, data analysis and data driven outcomes. Terabyte storage is now commonplace and available for less $\$ 150$. Furthermore mass data storage in cloud-based systems enables almost unlimited data to be stored safely.

Data analytics have improved too. Long gone is the reliance on descriptive statistics; this has been replaced with complex mathematical transformations such as Fourier transformations and Gaussian analyses. Complex mathematics are being used along with advanced statistical techniques that allow large volume regression analyses to be performed with multiple co-variables.

There has been advancement in the quality of the human-computer interface. Data display systems have been simplified to enable local field and health workers to understand outputs for metadata systems. An example is mapping of infectious disease outbreaks that enable fieldworkers to isolate high-risk individuals and protect uninfected individuals based on their geography. Also, there has been a cultural shift towards accepting the reports from metadata analyses so that large international agencies such as the WHO, UN and World Bank recognize the validity of metadata analytics and their applicability to world health.

\subsection{Wearable Technologies Can Be Easily Shipped and Distributed}

A fifth critical area that has enabled large-scale deployment of wearable biosen- 
sors has been through improved methods of distribution. The ability to tag and follow millions of individual sensors into the field previously required a large infrastructure. Modern distribution and tracking systems enable thousands of units to be tracked in the field with relative ease. Advancement of drone-based systems will further facilitate the distribution of wearable biosensors into remote and potentially dangerous regions.

\subsection{Financial Profitability}

There is profitability in wearables and so investors are willing to risk substantial amounts of money to advance these technologies. Although much of this investment is directed towards traditional business models in high-income countries, the spillover effect enables highly advanced technologies to be rapidly developed for secondary deployment in poverty dense regions.

There are a multitude of technological, cultural and financial reasons as to why the wearable technology pipeline is rapidly advancing both in terms of quality, price and distribution. The pace of these developments will continue and accelerate. There is a unique opportunity therefore to consider the mass deployment of wearable technologies to people who have previously not had access to advanced healthcare technology-the world's poor.

\section{Three Case Studies: Wearable Sensors in Poverty}

To illustrate the potential application of wearable technologies in impoverished areas we will examine three specific scenarios.

\subsection{Case Study One: Mexico City}

According to the World Bank

(http://povertydata.worldbank.org/poverty/country/MEX), approximately 53 million people in Mexico in 2014 were living in poverty-more than half the population.

Mexico, in 2015 had a total adult population of approximately 78 million people aged 20 to 79 years old. International Diabetes Foundation data demonstrated that, (http://www.idf.org/membership/nac/mexico), 14.7\% of these adults had Type II diabetes, meaning that in Mexico there are about 11.5 million cases of Type II diabetes and 76,000 deaths per year attributable to the disease. Furthermore, there are about 4 million people in Mexico who have Type II diabetes but do not know it and approximately one third of the Mexican population has prediabetes, which is an elevation in plasma glucose predisposing the individual to develop Type II diabetes within 10 years. It is estimated that the cost per person with diabetes to the Mexican healthcare system is $\$ 911$ /year and that the total health care costs associated with Type II diabetes approximate \$11 billion. It is important to appreciate that these are the medical treatment costs and do not take into account the fact that people with Type II diabetes are prone to premature cardiovascular disease, blindness and immobility-all of which can curtail life expectancy. The total national cost of Type II diabetes in Mexico 
(taking into account lost tax and earning dollars) runs to trillions of dollars. With this burden of human misery and healthcare cost it is important to examine nationwide solutions as has been highlighted by the national diabetes report issued by the Mexican government [5]

There are approximately 8.5 million people living in Mexico City; about 3 million of who have prediabetes. It is known that $6 \%$ weight loss can help prevent Type II diabetes, if this occurs with nutritional changes and improved physical activity [6]. It could be envisaged for city of this size that a single wearable sensor could be offered to all individuals at risk of Type II diabetes (for example, age greater than 40 , obesity, family heritage and/or a prior diagnosis of Type II diabetes). The sensor, using current technology, could include an intelligent triaxial accelerometer that adapts its response to the activity level of the individual plus a continuous glucose monitor. These devices could connect via Bluetooth to a series of 1000 Intervention Stations scattered across Mexico City for example at bus stops or in supermarkets. The Intervention Stations could include: 1) automated uploading of data from the wearable sensor when the person is nearby; 2) a weighing scale; 3 ) a tablet like interface and 4) printer. The interface would be the means of interacting with the individual and the method of gathering information and interlacing with a cloud-based Electronic Medical Record.

The proposed network of 3 million sensors and 1000 Intervention Stations across Mexico City would enable people to self-determine their risk of Type II diabetes (i.e.; whether they are likely to have prediabetes) via the tablet interface and if appropriate, receive a printed nutrition and activity prescription plus the wearable sensor. Individuals would then return to the Intervention Stations weekly for their sensor to be uploaded and the intervention plan to be modified and printed.

Even if this approach was only half as successful as published reports for this type of intervention [7] [8] [9] [10], 1 million people in Mexico City will be prevented from developing Type 2 Diabetes with a annual financial saving of $\$ 1$ billion (this does not take into account the benefits of preventing lost productivity and diabetes associated illnesses). At current market trends, it can be estimated that a wearable sensor could be produced at $\$ 10$ a unit and the intervention station at $\$ 150$ per unit. The cost of the intervention would therefore be approximately $\$ 33$ million. It is recognized that these estimates are crude however this illustration reflects that the financial Return on Investment exceeds $\$ 25$ for every $\$ 1$ invested.

When this type of diabetes prevention program is used in small groups for example in companies, the Return on Investment is approximately $\$ 10$ for every $\$ 1$ invested [8] [11]. As a general rule, mass-scaling further improves the relative Return on Investment. Thus, delivering a high-quality technology-driven program for preventing Type 2 Diabetes in Mexico City could be associated with substantial financial savings as well as improved public health-preventing $1 \mathrm{mil}-$ lion people from living with diabetes and its sequelae.

The deliverables for a system, such as the one described above, are even great- 
er than preventing Type 2 Diabetes in Mexico City. If such a system were in place, great opportunities would exist to understand how best to intervene to prevent Type II diabetes in large poverty-dense urban populations. The 1000 Intervention Stations described above would become hubs for vast amounts of population-based data. Detailed analyses could be performed to identify the key factors that contribute to best outcomes. Numerous secondary analyses could be performed as well, to understand the role of the social determinants of health, such as poverty, violence, education and healthcare access [12] [13] [14], on diabetes prevention. These types of analyses could be combined to create a cycle of continuous improvement in the program effectiveness. Furthermore, these types of analytics would enable diabetes prevention planning to go beyond Mexico to the many countries where needs are also great. It is not only the wearable technologies themselves that offer advances in population health but also the data they provide.

It might be thought that Mexico City with its diabetes rate of $14.7 \%$ is high (the USA has a diabetes rate of $10 \%$ ) and so this example reflects a relatively extreme situation. This is not the case; many Middle Eastern countries already have Type II diabetes rates of greater than $25 \%$ of the population [15] [16]. Population wide interventions to prevent and even self-treat diabetes is a world health necessity. The point made in this paper is that wearable sensors can be an integral part of that solution. Type 2 Diabetes and other non-communicable disease are viewed by WHO as a principal treat to health worldwide [12] [13] [17] [18] [19].

\subsection{Case Study Two: Kibera Slum Nairobi}

There are about $21 / 2$ million people living in slums in Nairobi in about 200 different settlements. This represents about $60 \%$ of the Nairobi population. About 500,000 people live in Kibera, the largest slum in Africa and one of the largest in the world.

Kibera is a sprawling mass of indistinct homes made from cardboard, corrugated iron and discarded wood. There are no specific streets or sewerage systems. Some electricity is available but often it is taken without permit off the main grid. Health care is principally provided by charitable services and is sparse. In Kibera there are multiple healthcare challenges; three examples include lack of clean water, the unpredictable geo-localization of people and HIV spread.

In Kibera, sewage comes from people living in temporary homes and drains directly into open ditches in the street. Inevitably, waterborne illnesses and diarrheal disease are commonplace. Attempts have been made to improve water quality in Kibera such as water purification systems and drinking water filters. However, waterborne fecal illnesses abound [20]. Where could wearable technology fit in? At the simplest level skin patches worn for weeks at a time could detect when a person develops fever. The same skin patch can detect hydration status. Thus when a person develops diarrheal disease these inexpensive adhe- 
sive skin patches (powered by NFC cell phone systems) could be used to identify people who are ill and monitor hydration with clean fluid. Another wearable could be used to monitor particulate matter in water and warn the user as to whether a water sample is clean to drink. Wearables directed at water safety are not only relevant to people living in slums but for all 783 million people who do not have access to clean water worldwide [21] [22] [23].

In Kibera, HIV is endemic although precise infection rates are not know because of HIV-associated stigma; HIV levels are at about $40 \%$ in those who agree to be tested and $14 \%$ among expectant mothers [24]. There is significant amount of prostitution where condom use is negotiable and the price for sexual intercourse approximates $\$ 0.3$. HIV treatment and prevention are sporadic and so HIV infection rates are expected to increase. HIV is not the only infectious disease of concern in Kibera; others include tuberculosis (TB), malaria, hepatitis and infection with soil borne pathogens [20] [25] [26] [27]. Wearable sensors have multiple potential roles in infectious disease. One example, fever patches, has already been discussed. Other skin patches have transdermal capability and can include ELISA technology that could enable early TB or HIV infections to be detected and treated early-this might prevent people from becoming chronically infected [28] [29]. At the very least, these types of wearables would enable disease outbreak clusters to be identified and quarantined. New wearable technologies can be incorporated into intra-vaginal rings that not only incorporate sensors but also can potentially deliver interventions against infectious agents and vaccines. The application of wearable technology to infectious disease in manifold spanning surveillance through treatment.

The physical infrastructure of Kibera is errant; streets and homes are temporary and so people do not have addresses. It has proven almost impossible to deliver and sustain an Electronic Medical Record within Kibera [30] because in addition illiteracy rates are high. In this instance, wearable technologies can serve as patient identifiers and link individuals (potentially via biomarkers) to their medical records and potential treatments. Should ethical considerations permit, wearable sensors can also serve as geographical identifiers so that individuals can be located to improve healthcare delivery. This could enable remote blood and environmental sensing to be linked to individualized treatment. Such wearable technologies would also facilitate healthcare mapping so that resource planning could be targeted by greatest need. An example would be where a network of geo-tagged fever patches indicates the outbreak of a spreading infectious disease enabling fieldworkers to quarantine the sick and prevent spread of a potentially harmful infection.

Wearables have many potential applications to help improve the health of slum dwellers. It is often overlooked as to how many people are indeed live in slums. According to the United Nations, 18\% of all urban housing units (125 million units) worldwide are nonpermanent structures. The World Bank, http://data.worldbank.org/indicator/EN.POP.SLUM.UR.ZS, reports that there is a long list of countries where more than half of the urban population lives in slums including, Bangladesh, Benin, Bhutan, Bolivia, Cambodia, Chad, Central 
Africa Republic, Democratic Republic of Congo, Uganda, Yemen, Zambia, and Kenya.

The health challenges faced by people living in slums are enormous and multiplexed [31]. Wearable sensors have many potential roles to help improve the health of these individuals.

\subsection{Case Study Three: The Refugee Population}

Refugees, fleeing from conflict or persecution, are protected under international law. According to the United Nations Refugee Agency (http://www.unhcr.org/), there are approximately 20,000,000 refugees in the world. Their physical displacement and transitory existence create unique health challenges that most medical systems cannot support.

Refugees particularly experience diseases associated with harsh climates, undernutrition, dental cares, mental illness, poor water sanitation, physical injury (e.g. from torture), infectious disease such as TB and worsening of pre-existing conditions such as diabetes [32] [33]. Not only is their health problems manifold but also, refugees are displaced from their health care system and past medical records. In addition there are the challenges associated with language, homelessness and culture that make providing healthcare even more complex.

Wearable technologies are particularly attractive in this population. Specifically the health assessment and disease treatment capabilities that wearable technologies will offer are especially useful as they travel with the person. A closed loop technology-driven health system will move with the refugee. International standards could enable cloud based medical records to be organized in a standard and easily translated format. Conceptually, a refugee could be traveling with their own comprehensive health assessment kit, data from which could be accessed through international aid agencies. Wearable drug delivery systems could be a component of the kit too, so that medication is released depending on the data acquired by the sensors. It may seem far-fetched to suggest that a refugee fleeing for his or her life might wear a comprehensive medical system linked to a cloud-based electronic medical record and drug delivery system. However, this concept is already under development in high-income countries; specifically, the closed loop artificial pancreas program for patients with Type 1 (juvenile) Diabetes [34] [35]. In the Type 1 Diabetes closed loop system there is a wearable transdermal glucose sensor, a wearable triaxial accelerometer, a wearable computer for data integration, wireless data coupling to the medical care team and medication delivery via a wearable pump that delivers the correct dose of insulin as determined by the central algorithm. The Type 1 Diabetes closed loop system represents a complete integrated sensing and drug delivery system albeit for a highly specific disease state. However, this demonstrates that it is feasible to build such wearable system for healthcare. These types of wearable healthcare systems could be given to refuges by relief agencies along with blankets, hydration, warmth and food. Wearable technologies will be able to help refugees receive a reasonable level of healthcare. 


\section{Challenges}

Having reviewed the accelerating development of wearable sensors and three specific regional health issues affecting the poor, consistent challenges emerge as to how to advance the deployment of wearable technologies in people living in poverty.

\subsection{The Scale of the Problem Is Great}

There is a great need to develop effective and scalable health solutions for the world's poor. It is estimated that half of the world's population lives in poverty [1] [2] [3] [4] many of whom might benefit from the type of solutions outlined in this paper. The reason for supporting health efforts to redress the health disparities associated with poverty is not purely humanitarian. People who are sick cannot work; health is one of the key determinants of helping people escape poverty. Furthermore, the 3 billion people who live in poverty represent a sizeable target population for business. The deployment of Wearable Technologies to poverty-dense regions is therefore, a substantial opportunity.

\subsection{Technology Dissemination Is the Principal Challenge}

The development of the technology either with respect to device design or data handling is not the principal problem. Far more the issue is getting the right technology to the people who need it. Wearable technology units can be successfully disseminated to hundreds of millions of people-as has been shown in the commercial space. In poverty dense areas, the challenges are greater and include: 1) low literacy rates; 2) the absence of a culture of "healthiness"; 3) oftentimes, a cultural mistrust of "technology"; 4) low health and technology literacy rates; 5) poorly developed understanding of how best to integrate data from wearable sensors into health programming and local health systems and 6) failure to appreciate the impact of the social determinates of health [36] such as violence, drug abuse and lack of healthcare systems. Such issues represent the principal challenges for exploiting the massive potential benefit that wearable sensors offer for improving human health.

\subsection{Ethical and Privacy Issues}

In high-income countries, individual privacy is prized and a series of personal protection policies continue to be enacted. The right of privacy for people living in poverty must be equally respected. There is an incorrect tendency to treat the privacy rights of people living in poverty as less important than those who are wealthy. This is illogical and wrong. Broad reaching ethical standards need to be agreed-upon internationally with respect to protecting the rights of all individuals regardless of financial status.

\subsection{Lack of Standardization in the Wearable Sensor Industry}

There is a lack of unifying standards in the wearable sensor industry. Standards 
are inadequate at multiple junctures such as with respect to: 1) data accrual protocols; 2) wireless data transmission protocols; 3) data storage and retrieval protocols; 4) data analytical procedures; 5) ethical/privacy standards (as noted above) and 6) standardized approaches for data representation. There is an urgent need, before the field expands further, to agree upon standardization protocols that will enable wearable technologies to be more easily integrated and their data analyzed and used.

\subsection{One Size Does Not Fit All}

The fundamental assumption when building mass-scalable health solutions is that people and their health needs are generalizable. There is, in fact, tremendous heterogeneity as to how illnesses effect people and how people respond to interventions [37]. It is seemingly impossible to individualize wearable-based health interventions for hundreds of millions of people. However, metadata analytics ironically has the potential to generate personalization algorithms. Such algorithms enable certain people to be targeted because for them, Medication X has better efficacy for malaria prevention. Similarly, algorithms can exclude other individuals from Medication $\mathrm{X}$ so that resources are not wasted. Through intelligent analysis, individualization strategies may be possible even in a mass markets. The analytical ability to individualize is a "value added" element of high-quality mass-scalable wearable technologies.

\subsection{Believe in the Possible}

The potential humanitarian value of mass scalable wearable health interventions is obvious. However, there is reticence in believing that such approaches are really mass scalable to help the world's poor. It is clear from the cell phone marketplace, and the move towards worldwide Internet access and the global availability of low cost smart phones that the mass deployment of wearable technologies is possible. Wearable technologies can be built in high volume and at low cost and the mathematical knowhow and the data analytics are in place. A decade ago it would never have been believed that hundreds of millions of people living in poverty would carry cell phone. I believe that within a decade, millions of people in poverty will be wearing health kits-health sensors combined with autonomous health intervention systems. Believe it too!

\section{Conclusions}

When one considers the scale of global poverty, the health needs of the world's poor and thus the scope of the opportunity, it is remarkable how little research has been conducted in this arena. Working out how best to deploy wearable technologies to the world's poor not only is of academic interest but of social significance too. A global research agenda is necessary to direct an organized approach.

We can design, invest in, build and deliver high-quality technology-driven wearable health solutions that will improve the lives of hundreds of millions of 
people especially those trapped in poverty. By doing so, we can improve world health and give millions of people an opportunity to escape the poverty trap.

\section{Acknowledgements}

This work was funded by NIH grants, R42 DK085738-02, 5R01DK085516-05 and R015R01HL114024-1 and a CODE Award from Mayo Clinic Center for Innovation.

\section{References}

[1] (2007) Tackling Global Poverty. Nature Nanotechnology, 2, 661. https://doi.org/10.1038/nnano.2007.349

[2] (2005) The State of the World's Children. The United Nations Children's Fund (UNICEF), 2004. New York.

[3] Dying for Change. Poor People's Experience of Health and Ill-Health, 2001. World Health Organization, Switzerland.

[4] Tollefson, J. (2015) Can Randomized Trials Eliminate Global Poverty? Nature, 524, 150-153. https://doi.org/10.1038/524150a

[5] Arredondo, A. (2016) Universal Coverage and Economic Burden from Epidemiological Changes of Diabetes in Latin America. Journal of Global Health, 6, Article ID: 020309. https://doi.org/10.7189/jogh.06.020309

[6] Eriksson, J., et al. (1999) Prevention of Type II Diabetes in Subjects with Impaired Glucose Tolerance: The Diabetes Prevention Study (DPS) in Finland. Study Design and 1-year Interim Report on the Feasibility of the Lifestyle Intervention Programme. Diabetologia, 42, 793-801. https://doi.org/10.1007/s001250051229

[7] Ackermann, R.T., et al. (2009) Changes in Health State Utilities with Changes in Body Mass in the Diabetes Prevention Program. Obesity (Silver Spring), 17, 2176 2181. https://doi.org/10.1038/oby.2009.114

[8] Ramachandran, A., et al. (2007) Cost-Effectiveness of the Interventions in the Primary Prevention of Diabetes among Asian Indians: Within-Trial Results of the Indian Diabetes Prevention Programme (IDPP). Diabetes Care, 30, 2548-2552. https://doi.org/10.2337/dc07-0150

[9] Sepah, S.C., Jiang, L. and Peters, A.L. (2014) Translating the Diabetes Prevention Program into an Online Social Network: Validation against CDC Standards. The Diabetes Educator, 40, 435-443. https://doi.org/10.1177/0145721714531339

[10] Taradash, J., Kramer, M., Molenaar, D., Arena, V., Vanderwood, K. and Kriska, A.M. (2015) Recruitment for a Diabetes Prevention Program Translation Effort in a Worksite Setting. Contemporary Clinical Trials, 41, 204-210. https://doi.org/10.1016/j.cct.2015.01.010

[11] Carroll, J., et al. (2015) Process Evaluation of Practice-Based Diabetes Prevention Programs: What Are the Implementation Challenges? The Diabetes Educator, 41, 271-279. https://doi.org/10.1177/0145721715572444

[12] World Health Organization Commission on the Social Determinants of Health (2008) Social Injustice Is Killing People on a Grand Scale. Scandinavian Journal of Public Health, 36, 896-897.

[13] Pratt, M., et al. (2012) The Implications of Megatrends in Information and Communication Technology and Transportation for Changes in Global Physical Activity. The Lancet, 380, 282-293. https://doi.org/10.1016/S0140-6736(12)60736-3 
[14] Sommer, I., et al. (2015) Socioeconomic Inequalities in Non-Communicable Diseases and Their Risk Factors: An Overview of Systematic Reviews. BMC Public Health, 15, 914. https://doi.org/10.1186/s12889-015-2227-y

[15] Chan, J.C., Zhang, Y. and Ning, G. (2014) Diabetes in China: A Societal Solution for a Personal Challenge. The Lancet Diabetes \& Endocrinology, 2, 969-979. https://doi.org/10.1016/S2213-8587(14)70144-5

[16] Peykari, N., et al. (2015) Diabetes Research in Middle East Countries: A Scientometrics Study from 1990 to 2012. Journal of Research in Medical Sciences, 20, 253-262.

[17] Levin, C. and Chisholm, D. (2016) Cost-Effectiveness and Affordability of Interventions, Policies, and Platforms for the Prevention and Treatment of Mental, Neurological, and Substance Use Disorders, in Mental, Neurological, and Substance Use Disorders. In: Patel, V., et al., Ed., Mental, Neurological, and Substance Use Disorders: Disease Control Priorities, 3d Edition, Vol. 4, The International Bank for Reconstruction and Development/The World Bank, Washington DC.

[18] Misra, A. and Khurana, L. (2008) Obesity and the Metabolic Syndrome in Developing Countries. The Journal of Clinical Endocrinology \& Metabolism, 93, S9-S30. https://doi.org/10.1210/jc.2008-1595

[19] World Health Organization (2003) WHO Mortality Database.

[20] Verani, J.R., et al. (2015) Burden of Invasive Nontyphoidal Salmonella Disease in a Rural and Urban Site in Kenya, 2009-2014. Clinical Infectious Diseases, 61, S302S309. https://doi.org/10.1093/cid/civ728

[21] Rush, E.C. (2013) Water: Neglected, Unappreciated and under Researched. European Journal of Clinical Nutrition, 67, 492-495. https://doi.org/10.1038/ejcn.2013.11

[22] Vorosmarty, C.J., Green, P., Salisbury, J. and Lammers, R.B. (2000) Global Water Resources: Vulnerability from Climate Change and Population Growth. Science, 289, 284-288. https://doi.org/10.1126/science.289.5477.284

[23] Watts, J. (2003) Forum Debates Private Sector Role in Global Water Supply. The Lancet, 361, 1022-1023. https://doi.org/10.1016/S0140-6736(03)12849-8

[24] Judd, M.C., et al. (2015) The Role of HIV in the Household Introduction and Transmission of Influenza in an Urban Slum, Nairobi, Kenya, 2008-2011. The Journal of Infectious Diseases, 212, 740-744. https://doi.org/10.1093/infdis/jiv106

[25] Davis, S.M., et al. (2014) Soil-Transmitted Helminths in Pre-School-Aged and School-Aged Children in an Urban Slum: A Cross-Sectional Study of Prevalence, Distribution, and Associated Exposures. The American Journal of Tropical Medicine and Hygiene, 91, 1002-1010. https://doi.org/10.4269/ajtmh.14-0060

[26] Furukawa, N.W., et al. (2016) Serologic Evidence for Hepatitis E Virus Infection among Patients with Undifferentiated Acute Febrile Illness in Kibera, Kenya. Journal of Clinical Virology, 77, 106-108. https://doi.org/10.1016/j.jcv.2016.02.021

[27] Wong, J.M., et al. (2015) Increased Rates of Respiratory and Diarrheal Illnesses in HIV-Negative Persons Living with HIV-Infected Individuals in a Densely Populated Urban Slum in Kenya. The Journal of Infectious Diseases, 212, 745-753. https://doi.org/10.1093/infdis/jiv107

[28] Katz, D.F., A. Yuan, and Y. Gao (2015) Vaginal Drug Distribution Modeling. Advanced Drug Delivery Reviews, 92, 2-13.

[29] Kiser, P.F., T.J. Johnson, and J.T. Clark (2012) State of the Art in Intravaginal Ring Technology for Topical Prophylaxis of HIV Infection. AIDS Reviews, 14, 62-77.

[30] Jawhari, B., et al. (2016) Barriers and Facilitators to Electronic Medical Record (EMR) Use in an Urban Slum. International Journal of Medical Informatics, 94, 246-254. https://doi.org/10.1016/j.ijmedinf.2016.07.015 
[31] Gopalan, A., et al. (2016) Health-Specific Information and Communication Technology Use and Its Relationship to Obesity in High-Poverty, Urban Communities: Analysis of a Population-Based Biosocial Survey. Journal of Medical Internet Research, 18, e182. https://doi.org/10.2196/jmir.5741

[32] De Smalen, A.W., Ghorab, H., Abd El Ghany, M. and Hill-Cawthorne, G.A. (2017) Refugees and Antimicrobial Resistance: A Systematic Review. Travel Medicine and Infectious Disease, 15, 23-28. https://doi.org/10.1016/j.tmaid.2016.12.001

[33] Sonne, C., Carlsson, J., Bech, P. and Mortensen, E.L. (2017) Pharmacological Treatment of Refugees with Trauma-Related Disorders: What Do We Know Today? Transcultural Psychiatry, 54, 260-280. https://doi.org/10.1177/1363461516682180

[34] Blauw, H., Keith-Hynes, P., Koops, R. and DeVries, J.H. (2016) A Review of Safety and Design Requirements of the Artificial Pancreas. Annals of Biomedical Engineering, 44, 3158-3172. https://doi.org/10.1007/s10439-016-1679-2

[35] Thabit, H. and R. Hovorka (2016) Coming of Age: The Artificial Pancreas for Type 1 Diabetes. Diabetologia, 59, 1795-1805. https://doi.org/10.1007/s00125-016-4022-4

[36] Markwick, A., Ansari, Z., Sullivan, M. and McNeil, J. (2014) Social Determinants and lifestyle Risk Factors Only Partially Explain the Higher Prevalence of Food Insecurity among Aboriginal and Torres Strait Islanders in the Australian State of Victoria: A Cross-Sectional Study. BMC Public Health, 14, 598. https://doi.org/10.1186/1471-2458-14-598

[37] Zahran, H.S., et al. (2005) Health-Related Quality of Life Surveillance-United States, 1993-2002. MMWR Surveillance Summaries, 54, 1-35.

Submit or recommend next manuscript to SCIRP and we will provide best service for you:

Accepting pre-submission inquiries through Email, Facebook, LinkedIn, Twitter, etc. A wide selection of journals (inclusive of 9 subjects, more than 200 journals) Providing 24-hour high-quality service User-friendly online submission system Fair and swift peer-review system Efficient typesetting and proofreading procedure Display of the result of downloads and visits, as well as the number of cited articles Maximum dissemination of your research work

Submit your manuscript at: http://papersubmission.scirp.org/ Or contact ti@scirp.org 